\title{
Well-posedness result for the Kuramoto-Velarde equation
}

\author{
Giuseppe Maria Coclite ${ }^{1}\left[\right.$ L $^{-}$Lorenzo di Ruvo $^{2}$
}

Received: 17 April 2021 / Accepted: 12 July 2021 / Published online: 20 July 2021

(c) The Author(s) 2021

\begin{abstract}
The Kuramoto-Velarde equation describes slow space-time variations of disturbances at interfaces, diffusion-reaction fronts and plasma instability fronts. It also describes BenardMarangoni cells that occur when there is large surface tension on the interface in a microgravity environment. Under appropriate assumption on the initial data, of the time $T$, and the coefficients of such equation, we prove the well-posedness of the classical solutions for the Cauchy problem, associated with this equation.
\end{abstract}

Keywords Existence $\cdot$ Uniqueness $\cdot$ Stability $\cdot$ Kuramoto-Velarde equation · Cauchy problem

Mathematics Subject Classification $35 \mathrm{G} 25 \cdot 35 \mathrm{~K} 55$

\section{Introduction}

In this paper, we investigate the well-posedness of the following Cauchy problem:

$$
\left\{\begin{array}{rlrl}
\partial_{t} u+\kappa u \partial_{x} u+v \partial_{x}^{2} u+\delta \partial_{x}^{3} u & & \\
& +\beta^{2} \partial_{x}^{4} u+\gamma\left(\partial_{x} u\right)^{2}+\alpha u \partial_{x}^{2} u=0, & & 0<t<T, \quad x \in \mathbb{R}, \\
u(0, x)=u_{0}(x), & & x \in \mathbb{R},
\end{array}\right.
$$

The authors are members of the Gruppo Nazionale per l'Analisi Matematica, la Probabilità e le loro Applicazioni (GNAMPA) of the Istituto Nazionale di Alta Matematica (INdAM). GMC has been partially supported by the Research Project of National Relevance "Multiscale Innovative Materials and Structures" Granted by the Italian Ministry of Education, University and Research (MIUR Prin 2017, Project Code 2017J4EAYB and the Italian Ministry of Education, University and Research under the Programme Department of Excellence Legge 232/2016 (Grant No. CUP - D94I18000260001).

$\bowtie$ Giuseppe Maria Coclite

giuseppemaria.coclite@poliba.it

Lorenzo di Ruvo

lorenzo.diruvo77@gmail.com

1 Dipartimento di Meccanica, Matematica e Management, Politecnico di Bari, Via E. Orabona 4, Bari 70125, Italy

2 Dipartimento di Matematica, Università di Bari, Via E. Orabona 4, Bari 70125, Italy 
with $\kappa, v, \delta, \beta, \gamma, \alpha \in \mathbb{R}$.

On the initial datum, we assume

$$
u_{0} \in H^{2}(\mathbb{R}), \quad u_{0} \neq 0
$$

and one of the following

$$
\begin{aligned}
& \beta \neq 0, \quad \beta^{2}>\frac{2 \tau^{2} T}{\log \left(A_{0}\right)}, \\
& \beta \neq 0, \quad 2\left\|u_{0}\right\|_{H^{1}(\mathbb{R})}^{2}+1<\sup _{\lambda \in\left(0, \frac{1}{4}\right)} \frac{\left(1+e^{\frac{4 \tau^{2} \sqrt{1-4 \lambda} T}{\beta^{2}}}\right) \sqrt{1-4 \lambda}}{e^{\frac{4 \tau^{2} \sqrt{1-4 \lambda} T}{\beta^{2}}}-1}, \\
& \beta \neq 0, \quad \beta^{2}>\left(2\left\|u_{0}\right\|_{H^{1}(\mathbb{R})}^{2}+1\right) \tau^{2} T, \\
& \beta \neq 0, \quad \beta^{2}>4 \tau^{2} T, \quad\left\|u_{0}\right\|_{H^{1}(\mathbb{R})}^{2} \leq \frac{-\tau^{2} T+\sqrt{\tau^{2} T \beta^{2}-3 \tau^{4} T^{2}}}{2 \tau^{2} T},
\end{aligned}
$$

where

$$
\tau^{2}=\max \left\{\kappa^{2}+\alpha^{2}, 2 v^{2}, \frac{3(\gamma-2 \alpha)^{2}}{4}, 1\right\} \neq 0, \quad A_{0}=\frac{\left\|u_{0}\right\|_{H^{1}(\mathbb{R})}^{2}+1}{\left\|u_{0}\right\|_{H^{1}(\mathbb{R})}^{2}} .
$$

From a physical point of view, Eq. (1.1), known as the Kuramoto-Velarde equation, describes slow space-time variations of disturbances at interfaces, diffusion-reaction fronts and plasma instability fronts [1-3]. It also describes Benard-Marangoni cells that occur when there is large surface tension on the interface [4-6] in a microgravity environment. This situation arises in crystal growth experiments aboard an orbiting space station, although the free interface is metastable with respect to small perturbations. In particular, the nonlinearities, $\gamma\left(\partial_{x} u\right)^{2}$ and $\alpha u \partial_{x}^{2} u$, model pressure destabilization effects striving to rupture the interface. Moreover, in [7], (1.1) is deduced to describe the long waves on a viscous fluid flowing down an inclined plane, while, in [8], (1.1) is deduced to model the drift waves in a plasma.

In [9-14] (1.1) is used to model the spinodal decomposition of phase separating systems in an external field, while, in [15-17], (1.1) is used to describe the spatiotemporal evolution of the morphology of steps on crystal surfaces. Finally, in [18-21], (1.1) is deduced to describe the growth of thermodynamically unstable crystal surfaces with strongly anisotropic surface tension.

From a mathematical point of view, in [22], the exact solutions for (1.1) are studied, while in [23], the initial boundary problem is analyzed. In [1,24], the existence of the solitons is proven, while in [25], the existence of traveling wave solutions for (1.1) is analyzed. In [26], the author analyzes the existence of the periodic solution for (1.1), under appropriate assumptions on $\kappa, v, \delta, \beta, \gamma, \alpha$. The well-posedness of the Cauchy problem for (1.1) is proven in [27], using the energy space technique and assuming $\kappa=0$, and in [28], through a priori estimates together with an application of the Cauchy-Kovalevskaya and choosing

$$
\gamma=2 \alpha
$$

In particular, in [27], the author gives some suitable conditions on $v, \delta, \beta, \gamma, \alpha$, and prove the local well-posedness of (1.1), with $\kappa=0$. Instead, in [28], under Assumptions (1.2) and (1.8), the authors prove well-posedness of (1.1), for each choose of $\beta$ and $T$. 
Observe that (1.1) generalizes the following equation:

$$
\partial_{t} u+\kappa u \partial_{x} u+v \partial_{x}^{2} u+\delta \partial_{x}^{3} u+\beta^{2} \partial_{x}^{4} u=0,
$$

that (1.9) was also independently deduced by Kuramoto [29-31] to describe the phase turbulence in reaction-diffusion systems, and by Sivashinsky [32] to describe plane flame propagation, taking into account the combined influence of diffusion and thermal conduction of the gas on the stability of a plane flame front.

Equation (1.9) can be used to study incipient instabilities in several physical and chemical systems [33-35]. Moreover, (1.9), which is also known as the Benney-Lin equation [36,37], was derived by Kuramoto in the study of phase turbulence in Belousov-Zhabotinsky reactions [38].

The dynamical properties and the existence of exact solutions for (1.9) have been investigated in [39-44]. In [45-47], the control problem for (1.9) with periodic boundary conditions, and on a bounded interval are studied, respectively. In [48], the problem of global exponential stabilization of (1.9) with periodic boundary conditions is analyzed. In [49], it is proposed a generalization of optimal control theory for (1.9), while in [50] the problem of global boundary control of (1.9) is considered. In [51], the existence of solitonic solutions for (1.9) is proven. In [28,52-54], the well-posedness of the Cauchy problem for (1.9) is proven, using the energy space technique, the fixed point method, a priori estimates together with an application of the Cauchy-Kovalevskaya Theorem and a priori estimates together with an application of the Aubin-Lions Lemma, respectively. Instead, in [55-57], the initial-boundary value problem for (1.1) is studied, using a priori estimates together with an application of the Cauchy-Kovalevskaya Theorem, and the energy space technique, respectively. Finally, following [58-60], in [61], the convergence of the solution of (1.9) to the unique entropy one of the Burgers equation is proven.

The main result of this paper is the following theorem.

Theorem 1.1 Assuming that (1.2) and one within (1.3), (1.4), (1.5), (1.6) hold, there exists a unique solution $u$ of (1.1), such that

$$
u \in H^{1}((0, T) \times \mathbb{R}) \cap L^{\infty}\left(0, T ; H^{2}(\mathbb{R})\right) .
$$

Moreover, if $u_{1}$ and $u_{2}$ are two solutions of (1.1) in correspondence of the initial data $u_{1,0}$ and $u_{2,0}$, we have that

$$
\left\|u_{1}(t, \cdot)-u_{2}(t, \cdot)\right\|_{L^{2}(\mathbb{R})} \leq e^{C t}\left\|u_{1,0}-u_{2,0}\right\|_{L^{2}(\mathbb{R})},
$$

for some suitable $C>0$, and every $0 \leq t \leq T$.

Compared to [27], Theorem 1.1 gives some conditions on $u_{0}, \beta$ and $T$ to have classical solutions for (1.1), under Assumption (1.2). Moreover, the argument of Theorem 1.1 relies on deriving suitable a priori estimates together with the existence result in [28].

The paper is organized as follows. In Sect. 2, we prove some a priori estimates of (1.1), under Assumptions (1.3), (1.4), (1.5) and (1.6), respectively. Those play a key role in the proof of our main result, which is given in Sect. 3 .

\section{A priori estimates}

In this section, we prove some a priori estimates on $u$.

We prove the following result. 
Lemma 2.1 We have that

$$
\frac{\|u(t, \cdot)\|_{H^{1}(\mathbb{R})}^{2}}{\|u(t, \cdot)\|_{H^{1}(\mathbb{R})}^{2}+1} \leq \frac{\left\|u_{0}\right\|_{H^{1}(\mathbb{R})}^{2}}{\left\|u_{0}\right\|_{H^{1}(\mathbb{R})}^{2}+1} e^{\frac{2 \tau^{2} t}{\beta^{2}}},
$$

for every $0 \leq t \leq T$, where $\tau^{2}$ is defined in (1.7). In particular, if (1.3) holds, there exists a constant $C>0$, such that

$$
\|u(t, \cdot)\|_{H^{1}(\mathbb{R})}^{2} \leq C,
$$

for every $0 \leq t \leq T$. Moreover,

$$
\begin{array}{r}
\|u\|_{L^{\infty}((0, T) \times \mathbb{R})} \leq C, \\
\int_{0}^{t}\left\|\partial_{x}^{2} u(s, \cdot)\right\|_{L^{2}(\mathbb{R})}^{2} d s \leq C, \\
\int_{0}^{t}\left\|\partial_{x}^{3} u(s, \cdot)\right\|_{L^{2}(\mathbb{R})}^{2} d s \leq C, \\
\int_{0}^{t}\left\|\partial_{x} u(s, \cdot)\right\|_{L^{4}(\mathbb{R})}^{4} d s \leq C,
\end{array}
$$

for every $0 \leq t \leq T$.

Proof Let $0 \leq t \leq T$ be given. Multiplying (1.1) by $2 u-2 \partial_{x}^{2} u$, an integration on $\mathbb{R}$ gives

$$
\begin{aligned}
\frac{d}{d t}(\| & \left.u(t, \cdot)\left\|_{L^{2}(\mathbb{R})}^{2}+\right\| \partial_{x} u(t, \cdot) \|_{L^{2}(\mathbb{R})}^{2}\right) \\
= & 2 \int_{\mathbb{R}} u \partial_{x} u d x-2 \int_{\mathbb{R}} \partial_{x}^{2} u \partial_{t} u d x \\
= & -2 \kappa \int_{\mathbb{R}} u^{2} \partial_{x} u d x+2 \kappa \int_{\mathbb{R}} u \partial_{x} u \partial_{x}^{2} u d x-2 v \int_{\mathbb{R}} u \partial_{x}^{2} u d x \\
& +2 v \int_{\mathbb{R}}\left(\partial_{x}^{2} u\right)^{2} d x-2 \delta \int_{\mathbb{R}} u \partial_{x}^{3} u d x+2 \delta \int_{\mathbb{R}} \partial_{x}^{2} u \partial_{x}^{3} u d x \\
& -2 \beta^{2} \int_{\mathbb{R}} u \partial_{x}^{4} u d x+2 \beta \int_{\mathbb{R}} \partial_{x}^{2} u \partial_{x}^{3} u d x-2 \gamma \int_{\mathbb{R}} u\left(\partial_{x} u\right)^{2} d x \\
& +2 \gamma \int_{\mathbb{R}}\left(\partial_{x} u\right)^{2} \partial_{x}^{2} u d x-2 \alpha \int_{\mathbb{R}} u^{2} \partial_{x}^{2} u d x+2 \alpha \int_{\mathbb{R}} u\left(\partial_{x}^{2} u\right)^{2} d x \\
= & 2 \kappa \int_{\mathbb{R}} u \partial_{x} u \partial_{x}^{2} u d x-2 v \int_{\mathbb{R}} u \partial_{x}^{2} u d x+2 v \int_{\mathbb{R}}\left(\partial_{x}^{2} u\right)^{2} d x \\
& +2 \delta \int_{\mathbb{R}} \partial_{x} u \partial_{x}^{2} u d x+2 \beta^{2} \int_{\mathbb{R}} \partial_{x} u \partial_{x}^{3} u d x-2 \beta^{2}\left\|\partial_{x}^{3} u(t, \cdot)\right\|_{L^{2}(\mathbb{R})}^{2} \\
& -2 \gamma \int_{\mathbb{R}} u\left(\partial_{x} u\right)^{2} d x-2 \alpha \int_{\mathbb{R}} u^{2} \partial_{x}^{2} u d x+2 \alpha \int_{\mathbb{R}} u\left(\partial_{x}^{2} u\right)^{2} d x \\
= & 2 \kappa \int_{\mathbb{R}} u \partial_{x} u \partial_{x}^{2} u d x-2 v \int_{\mathbb{R}} u \partial_{x}^{2} u d x+2 v \int_{\mathbb{R}}\left(\partial_{x}^{2} u\right)^{2} d x \\
& -2 \beta^{2}\left\|\partial_{x}^{2} u(t, \cdot)\right\|_{L^{2}(\mathbb{R})}^{2}-2 \beta^{2}\left\|\partial_{x}^{3} u(t, \cdot)\right\|_{L^{2}(\mathbb{R})}^{2}-2 \gamma \int_{\mathbb{R}} u\left(\partial_{x} u\right)^{2} d x \\
& -2 \alpha \int_{\mathbb{R}} u^{2} \partial_{x}^{2} u d x+2 \alpha \int_{\mathbb{R}} u\left(\partial_{x}^{2} u\right)^{2} d x .
\end{aligned}
$$


Therefore,

$$
\begin{aligned}
\frac{d}{d t}( & \left.\|u(t, \cdot)\|_{L^{2}(\mathbb{R})}^{2}+\left\|\partial_{x} u(t, \cdot)\right\|_{L^{2}(\mathbb{R})}^{2}\right) \\
& +2 \beta^{2}\left\|\partial_{x}^{2} u(t, \cdot)\right\|_{L^{2}(\mathbb{R})}^{2}+2 \beta^{2}\left\|\partial_{x}^{3} u(t, \cdot)\right\|_{L^{2}(\mathbb{R})}^{2} \\
= & 2 \kappa \int_{\mathbb{R}} u \partial_{x} u \partial_{x}^{2} u d x-2 v \int_{\mathbb{R}} u \partial_{x}^{2} u d x+2 v \int_{\mathbb{R}}\left(\partial_{x}^{2} u\right)^{2} d x \\
& -2 \gamma \int_{\mathbb{R}} u\left(\partial_{x} u\right)^{2} d x-2 \alpha \int_{\mathbb{R}} u^{2} \partial_{x}^{2} u d x+2 \alpha \int_{\mathbb{R}} u\left(\partial_{x}^{2} u\right)^{2} d x .
\end{aligned}
$$

Observe that

$$
\begin{gathered}
2 v \int_{\mathbb{R}}\left(\partial_{x}^{2} u\right)^{2} d x=2 v \int_{\mathbb{R}} \partial_{x}^{2} u \partial_{x}^{2} u d x=-2 v \int_{\mathbb{R}} \partial_{x} u \partial_{x}^{3} u d x, \\
2 \alpha \int_{\mathbb{R}} u\left(\partial_{x}^{2} u\right)^{2} d x=2 \alpha \int_{\mathbb{R}} u \partial_{x}^{2} u \partial_{x}^{2} u d x=-2 \alpha \int_{\mathbb{R}} u \partial_{x} u \partial_{x}^{3} u d x .
\end{gathered}
$$

Moreover,

$$
\begin{aligned}
\int_{\mathbb{R}} u\left(\partial_{x} u\right)^{2} d x & =\int_{\mathbb{R}} u \partial_{x} u \partial_{x} u d x=-\int_{\mathbb{R}} u \partial_{x}\left(u \partial_{x} u\right) d x \\
& =-\int_{\mathbb{R}} u\left(\partial_{x} u\right)^{2} d x-\int_{\mathbb{R}} u^{2} \partial_{x}^{2} u d x .
\end{aligned}
$$

Therefore,

$$
2 \int_{\mathbb{R}} u\left(\partial_{x} u\right)^{2} d x=-\int_{\mathbb{R}} u^{2} \partial_{x}^{2} u d x .
$$

Consequently, by (2.8) and (2.9), we have that

$$
\begin{aligned}
\frac{d}{d t}( & \left.\|u(t, \cdot)\|_{L^{2}(\mathbb{R})}^{2}+\left\|\partial_{x} u(t, \cdot)\right\|_{L^{2}(\mathbb{R})}^{2}\right) \\
& +2 \beta^{2}\left\|\partial_{x}^{2} u(t, \cdot)\right\|_{L^{2}(\mathbb{R})}^{2}+2 \beta^{2}\left\|\partial_{x}^{3} u(t, \cdot)\right\|_{L^{2}(\mathbb{R})}^{2} \\
= & 2 \kappa \int_{\mathbb{R}} u \partial_{x} u \partial_{x}^{2} u d x-2 v \int_{\mathbb{R}} u \partial_{x}^{2} u d x-2 v \int_{\mathbb{R}} \partial_{x} u \partial_{x}^{3} u d x \\
& +(\gamma-2 \alpha) \int_{\mathbb{R}} u^{2} \partial_{x}^{2} u d x-2 \alpha \int_{\mathbb{R}} u \partial_{x} u \partial_{x}^{3} u d x .
\end{aligned}
$$

Due to the Young inequality,

$$
\begin{aligned}
& 2|\kappa| \int_{\mathbb{R}}|u|\left|\partial_{x} u\right|\left|\partial_{x}^{2} u\right| d x=2 \int_{\mathbb{R}}\left|\frac{\kappa u \partial_{x} u}{\beta}\right|\left|\beta \partial_{x}^{2} u\right| d x \\
& \quad \leq \frac{\kappa^{2}}{\beta^{2}} \int_{\mathbb{R}} u^{2}\left(\partial_{x} u\right)^{2} d x+\beta^{2}\left\|\partial_{x}^{2} u(t, \cdot)\right\|_{L^{2}(\mathbb{R})}^{2} \\
& \quad \leq \frac{\kappa^{2}}{\beta^{2}}\|u(t, \cdot)\|_{L^{\infty}(\mathbb{R})}^{2}\left\|\partial_{x} u(t, \cdot)\right\|_{L^{2}(\mathbb{R})}^{2}+\beta^{2}\left\|\partial_{x}^{2} u(t, \cdot)\right\|_{L^{2}(\mathbb{R})}^{2}, \\
& 2|v| \int_{\mathbb{R}}|u|\left|\partial_{x}^{2} u\right| d x=2 \int_{\mathbb{R}}\left|\frac{v u}{\beta}\right|\left|\beta \partial_{x}^{2} u\right| d x \\
& \quad \leq \frac{2 v^{2}}{\beta^{2}}\|u(t, \cdot)\|_{L^{2}(\mathbb{R})}^{2}+\frac{\beta^{2}}{2}\left\|\partial_{x}^{2} u(t, \cdot)\right\|_{L^{2}(\mathbb{R})}^{2},
\end{aligned}
$$




$$
\begin{aligned}
2|v| & \int_{\mathbb{R}}\left|\partial_{x} u\right|\left|\partial_{x}^{3} u\right| d x=\int_{\mathbb{R}}\left|\frac{2 v \partial_{x} u}{\beta}\right|\left|\beta \partial_{x}^{3} u\right| d x \\
& \leq \frac{2 v^{2}}{\beta^{2}}\left\|\partial_{x} u(t, \cdot)\right\|_{L^{2}(\mathbb{R})}^{2}+\frac{\beta^{2}}{2}\left\|\partial_{x}^{3} u(t, \cdot)\right\|_{L^{2}(\mathbb{R})}^{2}, \\
\mid \gamma- & 2 \alpha\left|\int_{\mathbb{R}} u^{2}\right| \partial_{x}^{2} u\left|d x=2 \int_{\mathbb{R}}\right| \frac{\sqrt{3}(\gamma-2 \alpha) u^{2}}{2 \beta}|| \frac{\beta \partial_{x}^{2} u}{\sqrt{3}} \mid d x \\
& \leq \frac{3(\gamma-2 \alpha)^{2}}{4 \beta^{2}} \int_{\mathbb{R}} u^{4} d x+\frac{\beta^{2}}{3}\left\|\partial_{x}^{2} u(t, \cdot)\right\|_{L^{2}(\mathbb{R})}^{2} \\
& \leq \frac{3(\gamma-\alpha)^{2}}{4 \beta^{2}}\|u(t, \cdot)\|_{L^{\infty}(\mathbb{R})}^{2}\|u(t, \cdot)\|_{L^{2}(\mathbb{R})}^{2}+\frac{\beta^{2}}{3}\left\|\partial_{x}^{2} u(t, \cdot)\right\|_{L^{2}(\mathbb{R})}^{2}, \\
2|\alpha| & \int_{\mathbb{R}}\left|u \partial_{x} u \| \partial_{x}^{3} u\right| d x=2 \int_{\mathbb{R}}\left|\frac{\alpha u \partial_{x} u \mid}{\beta}\right|\left|\beta \partial_{x}^{3} u\right| d x \\
& \leq \frac{\alpha^{2}}{\beta^{2}} \int_{\mathbb{R}} u^{2}\left(\partial_{x} u\right)^{2} d x+\beta^{2}\left\|\partial_{x}^{3} u(t, \cdot)\right\|_{L^{2}(\mathbb{R})}^{2} \\
& \leq \frac{\alpha^{2}}{\beta^{2}}\|u(t, \cdot)\|_{L^{\infty}(\mathbb{R})}^{2}\left\|\partial_{x} u(t, \cdot)\right\|_{L^{2}(\mathbb{R})}^{2}+\beta^{2}\left\|\partial_{x}^{3} u(t, \cdot)\right\|_{L^{2}(\mathbb{R})}^{2} \cdot
\end{aligned}
$$

It follows from (1.3) and (2.7) that

$$
\begin{aligned}
\frac{d}{d t}(\| & \left.u(t, \cdot)\left\|_{L^{2}(\mathbb{R})}^{2}+\right\| \partial_{x} u(t, \cdot) \|_{L^{2}(\mathbb{R})}^{2}\right) \\
& +\frac{\beta^{2}}{6}\left\|\partial_{x}^{2} u(t, \cdot)\right\|_{L^{2}(\mathbb{R})}^{2}+\frac{\beta^{2}}{2}\left\|\partial_{x}^{3} u(t, \cdot)\right\|_{L^{2}(\mathbb{R})}^{2} \\
\leq & \frac{\kappa^{2}+\alpha^{2}}{\beta^{2}}\|u(t, \cdot)\|_{L^{\infty}(\mathbb{R})}^{2}\left\|\partial_{x} u(t, \cdot)\right\|_{L^{2}(\mathbb{R})}^{2} \\
& +\frac{2 v^{2}}{\beta^{2}}\left(\|u(t, \cdot)\|_{L^{2}(\mathbb{R})}^{2}+\left\|\partial_{x} u(t, \cdot)\right\|_{L^{2}(\mathbb{R})}^{2}\right) \\
& +\frac{3(\gamma-2 \alpha)^{2}}{4 \beta^{2}}\|u(t, \cdot)\|_{L^{\infty}(\mathbb{R})}^{2}\|u(t, \cdot)\|_{L^{2}(\mathbb{R})}^{2} \\
\leq & \frac{\kappa^{2}+\alpha^{2}}{\beta^{2}}\|u(t, \cdot)\|_{L^{\infty}(\mathbb{R})}^{2}\left(\|u(t, \cdot)\|_{L^{2}(\mathbb{R})}^{2}+\left\|\partial_{x} u(t, \cdot)\right\|_{L^{2}(\mathbb{R})}^{2}\right) \\
& +\frac{2 v^{2}}{\beta^{2}}\left(\|u(t, \cdot)\|_{L^{2}(\mathbb{R})}^{2}+\left\|\partial_{x} u(t, \cdot)\right\|_{L^{2}(\mathbb{R})}^{2}\right) \\
& +\frac{3(\gamma-2 \alpha)^{2}}{4 \beta^{2}}\|u(t, \cdot)\|_{L^{\infty}(\mathbb{R})}^{2}\left(\|u(t, \cdot)\|_{L^{2}(\mathbb{R})}^{2}+\left\|\partial_{x} u(t, \cdot)\right\|_{L^{2}(\mathbb{R})}^{2}\right) \\
& +\frac{1}{\beta^{2}}\left(\|u(t, \cdot)\|_{L^{2}(\mathbb{R})}^{2}+\left\|\partial_{x} u(t, \cdot)\right\|_{L^{2}(\mathbb{R})}^{2}\right) .
\end{aligned}
$$

Thanks to the Hölder inequality,

$$
u^{2}(t, x)=2 \int_{-\infty}^{x} u \partial_{x} u d x \leq 2 \int_{\mathbb{R}}\left|u\left\|\partial_{x} u \mid d x \leq 2\right\| u(t, \cdot)\left\|_{L^{2}(\mathbb{R})}\right\| \partial_{x} u(t, \cdot) \|_{L^{2}(\mathbb{R})} .\right.
$$

Hence,

$$
\|u(t, \cdot)\|_{L^{\infty}(\mathbb{R})}^{2} \leq 2\|u(t, \cdot)\|_{L^{2}(\mathbb{R})}\left\|\partial_{x} u(t, \cdot)\right\|_{L^{2}(\mathbb{R})} .
$$


Therefore, by the Young inequality,

$$
\|u(t, \cdot)\|_{L^{\infty}(\mathbb{R})}^{2} \leq\|u(t, \cdot)\|_{L^{2}(\mathbb{R})}^{2}+\left\|\partial_{x} u(t, \cdot)\right\|_{L^{2}(\mathbb{R})}^{2} .
$$

Consequently, by (2.11),

$$
\begin{aligned}
& \frac{d}{d t}\left(\|u(t, \cdot)\|_{L^{2}(\mathbb{R})}^{2}+\left\|\partial_{x} u(t, \cdot)\right\|_{L^{2}(\mathbb{R})}^{2}\right) \\
& \quad+\frac{\beta^{2}}{6}\left\|\partial_{x}^{2} u(t, \cdot)\right\|_{L^{2}(\mathbb{R})}^{2}+\frac{\beta^{2}}{2}\left\|\partial_{x}^{3} u(t, \cdot)\right\|_{L^{2}(\mathbb{R})}^{2} \\
& \leq \frac{\kappa^{2}+\alpha^{2}}{\beta^{2}}\left(\|u(t, \cdot)\|_{L^{2}(\mathbb{R})}^{2}+\left\|\partial_{x} u(t, \cdot)\right\|_{L^{2}(\mathbb{R})}^{2}\right)^{2} \\
& \quad+\frac{2 v^{2}}{\beta^{2}}\left(\|u(t, \cdot)\|_{L^{2}(\mathbb{R})}^{2}+\left\|\partial_{x} u(t, \cdot)\right\|_{L^{2}(\mathbb{R})}^{2}\right) \\
& \quad+\frac{3(\gamma-2 \alpha)^{2}}{\beta^{2}}\left(\|u(t, \cdot)\|_{L^{2}(\mathbb{R})}^{2}+\left\|\partial_{x} u(t, \cdot)\right\|_{L^{2}(\mathbb{R})}^{2}\right)^{2} \\
& \quad+\frac{1}{\beta^{2}}\left(\|u(t, \cdot)\|_{L^{2}(\mathbb{R})}^{2}+\left\|\partial_{x} u(t, \cdot)\right\|_{L^{2}(\mathbb{R})}^{2}\right) .
\end{aligned}
$$

We define the following function

$$
X(t):=\|u(t, \cdot)\|_{L^{2}(\mathbb{R})}^{2}+\left\|\partial_{x} u(t, \cdot)\right\|_{L^{2}(\mathbb{R})}^{2}=\|u(t, \cdot)\|_{H^{1}(\mathbb{R})}^{2}>0 .
$$

It follows from (1.7), (2.14) and (2.15) that

$$
\frac{d X(t)}{d t}+\frac{\beta^{2}}{6}\left\|\partial_{x}^{2} u(t, \cdot)\right\|_{L^{2}(\mathbb{R})}^{2}+\frac{\beta^{2}}{2}\left\|\partial_{x}^{3} u(t, \cdot)\right\|_{L^{2}(\mathbb{R})}^{2} \leq \frac{2 \tau^{2}}{\beta^{2}} X(t)(X(t)+1) .
$$

Therefore, we have that

$$
\frac{d X(t)}{d t} \leq \frac{2 \tau^{2}}{\beta^{2}} X(t)(X(t)+1)
$$

Consequently, by (2.15),

$$
\frac{1}{X(t)(X(t)+1)} \frac{d X(t)}{d t} \leq \frac{2 \tau^{2}}{\beta^{2}} .
$$

Integrating on $(0, t)$, we have that

$$
\log \left(\frac{X(t)}{X(t)+1}\right)-\log \left(\frac{X_{0}}{X_{0}+1}\right) \leq \frac{2 \tau^{2} t}{\beta^{2}} .
$$

Hence, by (1.2) and (2.15), we get

$$
\log \left(\frac{\|u(t, \cdot)\|_{H^{1}(\mathbb{R})}^{2}}{\|u(t, \cdot)\|_{H^{1}(\mathbb{R})}^{2}+1}\right) \leq \log \left(\frac{\left\|u_{0}\right\|_{H^{1}(\mathbb{R})}^{2}}{\left\|u_{0}\right\|_{H^{1}(\mathbb{R})}^{2}+1}\right)+\frac{2 \tau^{2} t}{\beta^{2}},
$$

which gives (2.1).

Assume (1.3) and we prove (2.2). We begin by observing that, by (2.1),

$$
\frac{\|u(t, \cdot)\|_{H^{1}(\mathbb{R})}^{2}}{\|u(t, \cdot)\|_{H^{1}(\mathbb{R})}^{2}+1} \leq \frac{\left\|u_{0}\right\|_{H^{1}(\mathbb{R})}^{2}}{\left\|u_{0}\right\|_{H^{1}(\mathbb{R})}^{2}+1} e^{\frac{2 \tau^{2} T}{\beta^{2}}} .
$$

We assume by contradiction that (2.2) does not hold, i.e.,

$$
\|u(t, \cdot)\|_{H^{1}(\mathbb{R})}^{2}=\infty .
$$


Therefore, by (2.18) and (2.19),

$$
\lim _{\|u(t, \cdot)\|_{H^{1}(\mathbb{R})}^{2} \rightarrow \infty} \frac{\|u(t, \cdot)\|_{H^{1}(\mathbb{R})}^{2}}{\|u(t, \cdot)\|_{H^{1}(\mathbb{R})}^{2}+1}=1 \leq \frac{\left\|u_{0}\right\|_{H^{1}(\mathbb{R})}^{2}}{\left\|u_{0}\right\|_{H^{1}(\mathbb{R})}^{2}+1} e^{\frac{2 \tau^{2} T}{\beta^{2}}} .
$$

It follows from (1.7) and (2.20) that

$$
\beta^{2} \leq \frac{2 \tau^{2} T}{\log \left(A_{0}\right)}
$$

which contradicts (1.3).

We prove (2.3). Due to (2.2) and (2.13), we have that

$$
\|u(t, \cdot)\|_{L^{\infty}(\mathbb{R})}^{2} \leq C .
$$

Hence,

$$
\|u\|_{L^{\infty}((0, T) \times \mathbb{R})}^{2} \leq C,
$$

which gives (2.3).

We prove (2.4) and (2.5). Thanks to (2.2) and (2.14),

$$
\begin{aligned}
& \frac{d}{d t}\left(\|u(t, \cdot)\|_{L^{2}(\mathbb{R})}^{2}+\left\|\partial_{x} u(t, \cdot)\right\|_{L^{2}(\mathbb{R})}^{2}\right) \\
& \quad+\frac{\beta^{2}}{6}\left\|\partial_{x}^{2} u(t, \cdot)\right\|_{L^{2}(\mathbb{R})}^{2}+\frac{\beta^{2}}{2}\left\|\partial_{x}^{3} u(t, \cdot)\right\|_{L^{2}(\mathbb{R})}^{2} \leq C .
\end{aligned}
$$

Integrating on $(0, t)$, by (1.2), we get

$$
\begin{aligned}
& \|u(t, \cdot)\|_{L^{2}(\mathbb{R})}^{2}+\left\|\partial_{x} u(t, \cdot)\right\|_{L^{2}(\mathbb{R})}^{2} \\
& \quad+\frac{\beta^{2}}{6} \int_{0}^{t}\left\|\partial_{x}^{2} u(s, \cdot)\right\|_{L^{2}(\mathbb{R})}^{2} d s+\frac{\beta^{2}}{2} \int_{0}^{t}\left\|\partial_{x}^{3} u(s, \cdot)\right\|_{L^{2}(\mathbb{R})}^{2} d s \\
& \quad \leq C_{0}+C t \leq C,
\end{aligned}
$$

which gives (2.4) and (2.5), respectively.

Finally, we prove (2.6). We begin by observing that [62] [Lemma 2.3] says that

$$
\left\|\partial_{x} u(t, \cdot)\right\|_{L^{4}(\mathbb{R})}^{4} \leq 6\left(\|u(t, \cdot)\|_{L^{2}(\mathbb{R})}^{2}+\left\|\partial_{x} u(t, \cdot)\right\|_{L^{2}(\mathbb{R})}^{2}\right)\left\|\partial_{x}^{2} u(t, \cdot)\right\|_{L^{2}(\mathbb{R})}^{2} .
$$

Consequently, by (2.2),

$$
\left\|\partial_{x} u(t, \cdot)\right\|_{L^{4}(\mathbb{R})}^{4} \leq C\left\|\partial_{x}^{2} u(t, \cdot)\right\|_{L^{2}(\mathbb{R})}^{2} .
$$

Integrating on $(0, t)$, by (2.4), we have (2.6).

Lemma 2.2 We have that

$$
\frac{2\|u(t, \cdot)\|_{H^{1}(\mathbb{R})}^{2}+1-\sqrt{1-4 \lambda}}{2\|u(t, \cdot)\|_{H^{1}(\mathbb{R})}^{2}+1+\sqrt{1-4 \lambda}} \leq \frac{2\left\|u_{0}\right\|_{H^{1}(\mathbb{R})}^{2}+1-\sqrt{1-4 \lambda}}{2\left\|u_{0}\right\|_{H^{1}(\mathbb{R})}^{2}+1+\sqrt{1-4 \lambda}} e^{\frac{4 \tau^{2} \sqrt{1-4 \lambda} t}{\beta^{2}}},
$$

for every $0 \leq t \leq T$ and some $\lambda \in(0,1 / 4)$. In particular, under Assumption (1.4), we have (2.2), (2.3), (2.4), (2.5) and (2.6). 
Proof Arguing as in Lemma 2.1, we have (2.17). Therefore, by (1.4), (1.7) and (2.17), we have that

$$
\frac{d X(t)}{d t} \leq \frac{2 \tau^{2}}{\beta^{2}} X(t)(X(t)+1) \leq \frac{2 \tau^{2}}{\beta^{2}}\left(X^{2}(t)+X(t)+\lambda\right) .
$$

Hence,

$$
\frac{1}{2\left(X^{2}(t)+X(t)+\lambda\right)} \frac{d X(t)}{d t} \leq \frac{\tau^{2}}{\beta^{2}}
$$

Thanks to (1.4) and (1.7), an integration on $(0, t)$ gives

$$
\log \left(\frac{2 X(t)+1-\sqrt{1-\lambda}}{2 X(t)+1+\sqrt{1-4 \lambda}}\right)-\log \left(\frac{2 X_{0}+1-\sqrt{1-4 \lambda}}{2 X_{0}+1+\sqrt{1-4 \lambda}}\right) \leq \frac{4 \tau^{2} \sqrt{1-4 \lambda} t}{\beta^{2}} .
$$

Consequently,

$$
\log \left(\frac{2 X(t)+1-\sqrt{1-4 \lambda}}{2 X(t)+1+\sqrt{1-4 \lambda}}\right) \leq \log \left(\frac{2 X_{0}+1-\sqrt{1-4 \lambda}}{2 X_{0}+1+\sqrt{1-4 \lambda}}\right)+\frac{4 \tau^{2} \sqrt{1-4 \lambda} t}{\beta^{2}} .
$$

(1.2), (2.15) and (2.22) give (2.21).

We assume (1.4) and we prove (2.2). We begin by observing that, by (2.21), we have that

$$
\frac{2\|u(t, \cdot)\|_{H^{1}(\mathbb{R})}^{2}+1-\sqrt{1-4 \lambda}}{2\|u(t, \cdot)\|_{H^{1}(\mathbb{R})}^{2}+1+\sqrt{1-4 \lambda}} \leq \frac{2\left\|u_{0}\right\|_{H^{1}(\mathbb{R})}^{2}+1-\sqrt{1-4 \lambda}}{2\left\|u_{0}\right\|_{H^{1}(\mathbb{R})}^{2}+1+\sqrt{1-4 \lambda}} e^{\frac{4 \tau^{2} \sqrt{1-4 \lambda} T}{\beta^{2}}} .
$$

We assume by contradiction that (2.2) does not hold, i.e., we have (2.19). Consequently, by (2.19) and (2.23),

$$
\begin{gathered}
\lim _{\|u(t, \cdot)\|_{H^{1}(\mathbb{R})}^{2} \rightarrow \infty} \frac{2\|u(t, \cdot)\|_{H^{1}(\mathbb{R})}^{2}+1-\sqrt{1-4 \lambda}}{2\|u(t, \cdot)\|_{H^{1}(\mathbb{R})}^{2}+1+\sqrt{1-4 \lambda}} \\
=1 \leq \frac{2\left\|u_{0}\right\|_{H^{1}(\mathbb{R})}^{2}+1-\sqrt{1-4 \lambda}}{2\left\|u_{0}\right\|_{H^{1}(\mathbb{R})}^{2}+1+\sqrt{1-4 \lambda}} e^{\frac{4 \tau^{2} \sqrt{1-4 \lambda} T}{\beta^{2}}} .
\end{gathered}
$$

Therefore, to (2.24), we have that

$$
2\left\|u_{0}\right\|_{H^{1}(\mathbb{R})}^{2}+1+\sqrt{1-4 \lambda} \leq\left(2\left\|u_{0}\right\|_{H^{1}(\mathbb{R})}^{2}+1-\sqrt{1-4 \lambda}\right) e^{\frac{4 \tau^{2} \sqrt{1-4 \lambda} T}{\beta^{2}}},
$$

that is

$$
\left(1-e^{\frac{4 \tau^{2} \sqrt{1-4 \lambda} T}{\beta^{2}}}\right)\left(2\left\|u_{0}\right\|_{H^{1}(\mathbb{R})}^{2}+1\right) \leq-\left(1+e^{\frac{4 \tau^{2} \sqrt{1-4 \lambda} T}{\beta^{2}}}\right) \sqrt{1-4 \lambda} .
$$

Hence,

$$
2\left\|u_{0}\right\|_{H^{1}(\mathbb{R})}^{2}+1 \geq \frac{\left(1+e^{\frac{4 \tau^{2} \sqrt{1-4 \lambda} T}{\beta^{2}}}\right) \sqrt{1-4 \lambda}}{e^{\frac{4 \tau^{2} \sqrt{1-4 \lambda} T}{\beta^{2}}}-1},
$$

which contradicts (1.4).

Finally, arguing as in Lemma 2.1, we have (2.3), (2.4), (2.5) and (2.6). 
Lemma 2.3 We have that

$$
\frac{-1}{2\|u(t, \cdot)\|_{H^{1}(\mathbb{R})}^{2}+1} \leq \frac{-1}{2\left\|u_{0}\right\|_{H^{1}(\mathbb{R})}^{2}+1}+\frac{\tau^{2} t}{\beta^{2}}
$$

for every $0 \leq t \leq T$. In particular, if (1.5) holds, we have (2.2), (2.3), (2.4), (2.5) and (2.6).

Proof Arguing as in Lemma 2.1, we have (2.17). Therefore, by (2.17), we have that

$$
\frac{d X(t)}{d t} \leq \frac{2 \tau^{2}}{\beta^{2}}\left(X^{2}(t)+X(t)+\frac{1}{4}\right)=\frac{\tau^{2}}{2 \beta^{2}}(2 X(t)+1)^{2} .
$$

Therefore,

$$
\frac{2}{(2 X(t)+1)^{2}} \frac{d X(t)}{d t} \leq \frac{\tau^{2}}{\beta^{2}} .
$$

Integrating on $(0, t)$, we have that

$$
\frac{-1}{2 X(t)+1}+\frac{1}{2 X_{0}+1} \leq \frac{\tau^{2} t}{\beta^{2}},
$$

that is,

$$
\frac{-1}{2 X(t)+1} \leq \frac{-1}{2 X_{0}+1}+\frac{\tau^{2} t}{\beta^{2}}
$$

(2.25) follows from (1.2) and (2.15).

Assume (1.5) and we prove (2.2). We begin by observing that, by (2.25), we have that

$$
\frac{-1}{2\|u(t, \cdot)\|_{H^{1}(\mathbb{R})}^{2}+1} \leq \frac{-1}{2\left\|u_{0}\right\|_{H^{1}(\mathbb{R})}^{2}+1}+\frac{\tau^{2} T}{\beta^{2}} .
$$

We assume by contradiction that (2.2) does not hold, i.e., we have (2.19). It follows from (2.19) and (2.27) that

$$
\lim _{\|u(t, \cdot)\|_{H^{1}(\mathbb{R})}^{2} \rightarrow \infty} \frac{-1}{2\|u(t, \cdot)\|_{H^{1}(\mathbb{R})}^{2}+1}=0 \leq \frac{-1}{2\left\|u_{0}\right\|_{H^{1}(\mathbb{R})}^{2}+1}+\frac{\tau^{2} T}{\beta^{2}} .
$$

Consequently, by (2.28), we have that

$$
\beta^{2} \leq\left(2\left\|u_{0}\right\|_{H^{1}(\mathbb{R})}^{2}+1\right) \tau^{2} T,
$$

which contradicts (1.5).

Finally, arguing as in Lemma 2.1, we have (2.3), (2.4), (2.5) and (2.6).

Lemma 2.4 We have that

$$
\arctan \left(\frac{2\|u(t, \cdot)\|_{H^{1}(\mathbb{R})}^{2}+1}{\sqrt{4 \lambda-1}}\right) \leq \arctan \left(\frac{2\left\|u_{0}\right\|_{H^{1}(\mathbb{R})}^{2}+1}{\sqrt{4 \lambda-1}}\right)+\frac{\tau^{2} \sqrt{4 \lambda-1} t}{\beta^{2}},
$$

for every $0 \leq t \leq T$, where

$$
\lambda>\frac{1}{4} .
$$

In particular, assuming (1.6) and taking

$$
\lambda \in\left(\frac{1}{4}, \frac{\beta^{2}-4 \tau^{2} T\left\|u_{0}\right\|_{H^{1}(\mathbb{R})}^{4}-4 \tau^{2} T\left\|u_{0}\right\|_{H^{1}(\mathbb{R})}^{2}}{4 \tau^{2} T}\right),
$$


we obtain the following inequality

$$
\frac{2\|u(t, \cdot)\|_{H^{1}(\mathbb{R})}^{2}+1}{\sqrt{4 \lambda-1}} \leq \tan \left(\arctan \left(\frac{2\left\|u_{0}\right\|_{H^{1}(\mathbb{R})}^{2}+1}{\sqrt{4 \lambda-1}}\right)+\frac{2 \tau^{2} \sqrt{4 \lambda-1} T}{\beta^{2}}\right),
$$

where

$$
\arctan \left(\frac{2\left\|u_{0}\right\|_{H^{1}(\mathbb{R})}^{2}+1}{\sqrt{4 \lambda-1}}\right)+\frac{2 \tau^{2} \sqrt{4 \lambda-1} T}{\beta^{2}} \in\left(0, \frac{\pi}{2}\right) .
$$

Moreover, (2.2), (2.3), (2.4), (2.5) and (2.6), hold.

Proof Arguing as in Lemma 2.1, we have (2.17). Therefore, by (2.17) and (2.30), we have that

$$
\frac{d X(t)}{d t} \leq \frac{2 \tau^{2}}{\beta^{2}}\left(X^{2}(t)+X(t)\right) \leq \frac{2 \tau^{2}}{\beta^{2}}\left(X^{2}(t)+X(t)+\lambda\right) .
$$

Hence,

$$
\frac{1}{X^{2}(t)+X(t)+\lambda} \frac{d X(t)}{d t} \leq \frac{2 \tau^{2}}{\beta^{2}} .
$$

Integrating on $(0, t)$, we have that

$$
\frac{2}{\sqrt{4 \lambda-1}} \arctan \left(\frac{2 X(t)+1}{\sqrt{4 \lambda-1}}\right)-\frac{2}{\sqrt{4 \lambda-1}} \arctan \left(\frac{2 X_{0}+1}{\sqrt{4 \lambda-1}}\right) \leq \frac{2 \tau^{2} t}{\beta^{2}}
$$

Hence,

$$
\arctan \left(\frac{2 X(t)+1}{\sqrt{4 \lambda-1}}\right) \leq \arctan \left(\frac{2 X_{0}+1}{\sqrt{4 \lambda-1}}\right)+\frac{\tau^{2} \sqrt{4 \lambda-1} t}{\beta^{2}} .
$$

(1.2), (2.15) and (2.34) give (2.29).

Assume (1.6) and we prove (2.32). We begin by observe that, by (2.29),

$$
\arctan \left(\frac{2\|u(t, \cdot)\|_{H^{1}(\mathbb{R})}^{2}+1}{\sqrt{4 \lambda-1}}\right) \leq \arctan \left(\frac{2\left\|u_{0}\right\|_{H^{1}(\mathbb{R})}^{2}+1}{\sqrt{4 \lambda-1}}\right)+\frac{\tau^{2} \sqrt{4 \lambda-1} T}{\beta^{2}} .
$$

We assume by contradiction that (2.2) does not hold, i.e., we have (2.19). It follows from (2.19) and (2.35) that

$$
\begin{aligned}
& \lim _{\|u(t, \cdot)\|_{H^{1}(\mathbb{R})}^{2} \rightarrow \infty} \arctan \left(\frac{2\|u(t, \cdot)\|_{H^{1}(\mathbb{R})}^{2}+1}{\sqrt{4 \lambda-1}}\right) \\
& =\frac{\pi}{2} \leq \arctan \left(\frac{2\left\|u_{0}\right\|_{H^{1}(\mathbb{R})}^{2}+1}{\sqrt{4 \lambda-1}}\right)+\frac{\tau^{2} \sqrt{4 \lambda-1} T}{\beta^{2}} .
\end{aligned}
$$

Therefore, we obtain that

$$
\frac{\pi}{2}-\arctan \left(\frac{2\left\|u_{0}\right\|_{H^{1}(\mathbb{R})}^{2}+1}{\sqrt{4 \lambda-1}}\right)-\frac{\tau^{2} \sqrt{4 \lambda-1} T}{\beta^{2}} \leq 0,
$$

that is

$$
\arctan \left(\frac{2\left\|u_{0}\right\|_{H^{1}(\mathbb{R})}^{2}+1}{\sqrt{4 \lambda-1}}\right)+\frac{\tau^{2} \sqrt{4 \lambda-1} T}{\beta^{2}}-\frac{\pi}{2} \geq 0
$$


We consider the following function

$$
F(\lambda)=\arctan \left(\frac{2\left\|u_{0}\right\|_{H^{1}(\mathbb{R})}^{2}+1}{\sqrt{4 \lambda-1}}\right)+\frac{\tau^{2} \sqrt{4 \lambda-1} T}{\beta^{2}}-\frac{\pi}{2},
$$

with

$$
\lambda \in\left(\frac{1}{4}, \infty\right)
$$

Observe that, by (2.38) and (2.39),

$$
\begin{aligned}
& \lim _{\lambda \rightarrow \frac{1}{4}^{+}} F(\lambda)=\lim _{\lambda \rightarrow \frac{1}{4}^{+}} \arctan \left(\frac{2\left\|u_{0}\right\|_{H^{1}(\mathbb{R})}^{2}+1}{\sqrt{4 \lambda-1}}\right)+\frac{\tau^{2} \sqrt{4 \lambda-1} T}{\beta^{2}}-\frac{\pi}{2}=0, \\
& \lim _{\lambda \rightarrow \infty} F(\lambda)=\lim _{\lambda \rightarrow \infty} \arctan \left(\frac{2\left\|u_{0}\right\|_{H^{1}(\mathbb{R})}^{2}+1}{\sqrt{4 \lambda-1}}\right)+\frac{\tau^{2} \sqrt{4 \lambda-1} T}{\beta^{2}}-\frac{\pi}{2}=\infty .
\end{aligned}
$$

Consequently, by (2.38) and (2.40), (2.37) is verified if

$$
F^{\prime}(\lambda)>0 \text { for } \lambda>\frac{1}{4} .
$$

Observe that, by (2.38),

$$
\begin{aligned}
F^{\prime}(\lambda) & =\frac{-1}{\frac{\left(2\left\|u_{0}\right\|_{H^{1}(\mathbb{R})}^{2}+1\right)^{2}}{4 \lambda-1}+1} \frac{1}{4 \lambda-1} \frac{2}{\sqrt{4 \lambda-1}}+\frac{\tau^{2} T}{\beta^{2}} \frac{2}{\sqrt{4 \lambda-1}} \\
& =\frac{-1}{4\left\|u_{0}\right\|_{H^{1}(\mathbb{R})}^{2}\left(\left\|u_{0}\right\|_{H^{1}(\mathbb{R})}^{2}+1\right)+4 \lambda} \frac{2}{\sqrt{4 \lambda-1}}+\frac{\tau^{2} T}{\beta^{2}} \frac{2}{\sqrt{4 \lambda-1}} .
\end{aligned}
$$

Hence, $F^{\prime}(\lambda)>0$ if and only if

$$
\frac{-1}{4\left\|u_{0}\right\|_{H^{1}(\mathbb{R})}^{2}\left(\left\|u_{0}\right\|_{H^{1}(\mathbb{R})}^{2}+1\right)+4 \lambda} \frac{2}{\sqrt{4 \lambda-1}}+\frac{\tau^{2} T}{\beta^{2}} \frac{2}{\sqrt{4 \lambda-1}} \geq 0
$$

that is

$$
4 \tau^{2} T \lambda+4 \tau^{2} T\left\|u_{0}\right\|_{H^{1}(\mathbb{R})}^{4}+4 \tau^{2} T\left\|u_{0}\right\|_{H^{1}(\mathbb{R})}^{2}-\beta^{2} \geq 0 .
$$

Thanks to (2.39), (2.43) is verified when

$$
4 \tau^{2} T\left\|u_{0}\right\|_{H^{1}(\mathbb{R})}^{4}+4 \tau^{2} T\left\|u_{0}\right\|_{H^{1}(\mathbb{R})}^{2}-\beta^{2} \geq 0,
$$

that is

$$
\left\|u_{0}\right\|_{H^{1}(\mathbb{R})}^{2} \geq \frac{-\tau^{2} T+\sqrt{\tau^{4} T^{2}+\tau^{2} T \beta^{2}}}{2 \tau^{2} T}
$$

which contradicts (1.6).

Therefore, if we assume (1.6), (2.37) cannot hold. Observe that, by (2.42), $F^{\prime}(\lambda) \leq 0$ when $\lambda>\frac{1}{4}$, if and only if,

$$
4 \tau^{2} T \lambda+4 \tau^{2} T\left\|u_{0}\right\|_{H^{1}(\mathbb{R})}^{4}+4 \tau^{2} T\left\|u_{0}\right\|_{H^{1}(\mathbb{R})}^{2}-\beta^{2} \leq 0,
$$

that is

$$
\lambda \leq \frac{\beta^{2}-4 \tau^{2} T\left\|u_{0}\right\|_{H^{1}(\mathbb{R})}^{4}-4 \tau^{2} T\left\|u_{0}\right\|_{H^{1}(\mathbb{R})}^{2}}{4 \tau^{2} T} .
$$


It follows from (2.30) and (2.44) that

$$
\frac{1}{4}<\lambda \leq \frac{\beta^{2}-4 \tau^{2} T\left\|u_{0}\right\|_{H^{1}(\mathbb{R})}^{4}-4 \tau^{2} T\left\|u_{0}\right\|_{H^{1}(\mathbb{R})}^{2}}{4 \tau^{2} T} .
$$

$\lambda$ exist, if

$$
\frac{1}{4}<\frac{\beta^{2}-4 \tau^{2} T\left\|u_{0}\right\|_{H^{1}(\mathbb{R})}^{4}-4 \tau^{2} T\left\|u_{0}\right\|_{H^{1}(\mathbb{R})}^{2}}{4 \tau^{2} T} .
$$

Hence,

$$
4 \tau^{2} T<\beta^{2}-4 \tau^{2} T\left\|u_{0}\right\|_{H^{1}(\mathbb{R})}^{4}-4 \tau^{2} T\left\|u_{0}\right\|_{H^{1}(\mathbb{R})}^{2},
$$

that is

$$
4 \tau^{2} T\left\|u_{0}\right\|_{H^{1}(\mathbb{R})}^{4}+4 \tau^{2} T\left\|u_{0}\right\|_{H^{1}(\mathbb{R})}^{2}+4 \tau^{2} T-\beta^{2} \leq 0,
$$

which is guaranteed by Assumption (1.6). Therefore, (2.32) holds.

Finally, thanks to (2.32) and (2.33), we have (2.2), while arguing as in Lemma 2.1, we have (2.3), (2.4), (2.5) and (2.6).

Lemma 2.5 Assume that one within (1.3), (1.4), (1.5), (1.6) holds. There exist a constant $C>0$, such that

$$
\left\|\partial_{x}^{2} u(t, \cdot)\right\|_{L^{2}(\mathbb{R})}^{2}+\beta^{2} \int_{0}^{t}\left\|\partial_{x}^{4} u(s, \cdot)\right\|_{L^{2}(\mathbb{R})}^{2} d s \leq C,
$$

for every $0 \leq t \leq T$. In particular,

$$
\left\|\partial_{x} u\right\|_{L^{\infty}((0, T) \times \mathbb{R})} \leq C .
$$

Proof Let $0 \leq t \leq T$. Multiplying (1.1) by $2 \partial_{x}^{4} u$, an integration on $\mathbb{R}$ gives

$$
\begin{aligned}
\frac{d}{d t}\left\|\partial_{x}^{2} u(t, \cdot)\right\|_{L^{2}(\mathbb{R})}^{2}= & 2 \int_{\mathbb{R}} \partial_{x}^{4} u \partial_{t} u d x \\
= & -2 \kappa \int_{\mathbb{R}} u \partial_{x} u \partial_{x}^{4} u d x-2 v \int_{\mathbb{R}} \partial_{x}^{2} u \partial_{x}^{4} u d x-2 \beta^{2}\left\|\partial_{x}^{4} u(t, \cdot)\right\|_{L^{2}(\mathbb{R})}^{2} \\
& +2 \delta \int_{\mathbb{R}} \partial_{x}^{3} u \partial_{x}^{4} u d x-2 \gamma \int_{\mathbb{R}}\left(\partial_{x} u\right)^{2} \partial_{x}^{4} u d x-2 \alpha \int_{\mathbb{R}} u \partial_{x}^{2} u \partial_{x}^{4} u d x \\
= & 2 \kappa \int_{\mathbb{R}}\left(\partial_{x} u\right)^{2} \partial_{x}^{3} u d x+2 \kappa \int_{\mathbb{R}} u \partial_{x}^{2} u \partial_{x}^{3} u d x+2 v\left\|\partial_{x}^{3} u(t, \cdot)\right\|_{L^{2}(\mathbb{R})}^{2} \\
& -2 \beta^{2}\left\|\partial_{x}^{4} u(t, \cdot)\right\|_{L^{2}(\mathbb{R})}^{2}-2 \gamma \int_{\mathbb{R}}\left(\partial_{x} u\right)^{2} \partial_{x}^{4} u d x-2 \alpha \int_{\mathbb{R}} u \partial_{x}^{2} u \partial_{x}^{4} u d x .
\end{aligned}
$$

Therefore, we have that

$$
\begin{aligned}
\frac{d}{d t}\left\|\partial_{x}^{2} u(t, \cdot)\right\|_{L^{2}(\mathbb{R})}^{2} & +\beta^{2}\left\|\partial_{x}^{4} u(t, \cdot)\right\|_{L^{2}(\mathbb{R})}^{2} \\
= & 2 \kappa \int_{\mathbb{R}}\left(\partial_{x} u\right)^{2} \partial_{x}^{3} u d x+2 \kappa \int_{\mathbb{R}} u \partial_{x}^{2} u \partial_{x}^{3} u d x-2 v\left\|\partial_{x}^{3} u(t, \cdot)\right\|_{L^{2}(\mathbb{R})}^{2} \\
& -2 \gamma \int_{\mathbb{R}}\left(\partial_{x} u\right)^{2} \partial_{x}^{4} u d x-2 \alpha \int_{\mathbb{R}} u \partial_{x}^{2} u \partial_{x}^{4} u d x .
\end{aligned}
$$

Due to (2.3) and the Young inequality,

$$
2\left|\kappa \int_{\mathbb{R}}\left(\partial_{x} u\right)^{2}\right| \partial_{x}^{3} u \mid d x \leq \kappa^{2}\left\|\partial_{x} u(t, \cdot)\right\|_{L^{4}(\mathbb{R})}^{4}+\left\|\partial_{x}^{3} u(t, \cdot)\right\|_{L^{2}(\mathbb{R})}^{2},
$$




$$
\begin{aligned}
& 2|\kappa| \int_{\mathbb{R}}\left|u\left\|\partial_{x}^{2} u\right\| \partial_{x}^{3} u\right| d x \leq 2|\kappa|\|u\|_{L^{\infty}((0, T) \times \mathbb{R})} \int_{\mathbb{R}}\left|\partial_{x}^{2} u \| \partial_{x}^{3} u\right| d x \\
& \quad \leq 2 C \int_{\mathbb{R}}\left|\partial_{x}^{2} u\left\|\partial_{x}^{3} u \mid d x \leq C\right\| \partial_{x}^{2} u(t, \cdot)\left\|_{L^{2}(\mathbb{R})}^{2}+C\right\| \partial_{x}^{3} u(t, \cdot) \|_{L^{2}(\mathbb{R})}^{2},\right. \\
& 2|\gamma| \int_{\mathbb{R}}\left(\partial_{x} u\right)^{2}\left|\partial_{x}^{4} u\right| d x=2 \int_{\mathbb{R}}\left|\frac{\gamma\left(\partial_{x} u\right)^{2}}{\beta}\right|\left|\beta \partial_{x}^{4} u\right| d x \\
& \quad \leq \frac{\gamma^{2}}{\beta^{2}}\left\|\partial_{x} u(t, \cdot)\right\|_{L^{4}(\mathbb{R})}^{4}+\beta^{2}\left\|\partial_{x}^{4} u(t, \cdot)\right\|_{L^{2}(\mathbb{R})}^{2}, \\
& 2|\alpha| \int_{\mathbb{R}}\left|u\left\|\partial_{x}^{2} u\right\| \partial_{x}^{4} u\right| d x \leq 2|\alpha|\|u\|_{L^{\infty}((0, T) \times \mathbb{R})} \int_{\mathbb{R}}\left|\partial_{x}^{2} u \| \partial_{x}^{4} u\right| d x \\
& \quad \leq C \int_{\mathbb{R}}\left|\partial_{x}^{2} u \| \partial_{x}^{4} u\right| d x=\int_{\mathbb{R}}\left|\frac{C \partial_{x}^{2} u}{\beta}\right|\left|\beta \partial_{x}^{4} u\right| d x \\
& \quad \leq C\left\|\partial_{x}^{2} u(t, \cdot)\right\|_{L^{2}(\mathbb{R})}^{2}+\frac{\beta^{2}}{2}\left\|\partial_{x}^{4} u(t, \cdot)\right\|_{L^{2}(\mathbb{R})}^{2} \cdot
\end{aligned}
$$

It follows from (2.47) that

$$
\begin{aligned}
& \frac{d}{d t}\left\|\partial_{x}^{2} u(t, \cdot)\right\|_{L^{2}(\mathbb{R})}^{2}+\frac{\beta^{2}}{2}\left\|\partial_{x}^{4} u(t, \cdot)\right\|_{L^{2}(\mathbb{R})}^{2} \\
& \quad \leq\left(\kappa^{2}+\frac{\gamma^{2}}{\beta^{2}}\right)\left\|\partial_{x} u(t, \cdot)\right\|_{L^{4}(\mathbb{R})}^{4}+C\left\|\partial_{x}^{3} u(t, \cdot)\right\|_{L^{2}(\mathbb{R})}^{2}+C\left\|\partial_{x}^{2} u(t, \cdot)\right\|_{L^{2}(\mathbb{R})}^{2} .
\end{aligned}
$$

Integrating on $(0, t)$, by (1.2), (2.4), (2.5) and (2.6), we get

$$
\begin{aligned}
\left\|\partial_{x}^{2} u(t, \cdot)\right\|_{L^{2}(\mathbb{R})}^{2} & +\frac{\beta^{2}}{2} \int_{0}^{t}\left\|\partial_{x}^{4} u(s, \cdot)\right\|_{L^{2}(\mathbb{R})}^{2} d s \\
\leq & C_{0}+\left(\kappa^{2}+\frac{\gamma^{2}}{\beta^{2}}\right) \int_{0}^{t}\left\|\partial_{x} u(s, \cdot)\right\|_{L^{4}(\mathbb{R})}^{4} d s \\
& +C \int_{0}^{t}\left\|\partial_{x}^{3} u(s, \cdot)\right\|_{L^{2}(\mathbb{R})}^{2} d s+C \int_{0}^{t}\left\|\partial_{x}^{2} u(s, \cdot)\right\|_{L^{2}(\mathbb{R})}^{2} d s \\
\leq & C_{0}+C \leq C,
\end{aligned}
$$

which gives (2.45).

Finally, we prove (2.46). Thanks to (2.2), (2.45) and the Hölder inequality,

$$
\begin{aligned}
\left(\partial_{x} u(t, x)\right)^{2} & =2 \int_{-\infty}^{x} \partial_{x} u \partial_{x}^{2} u d y \leq 2 \int_{\mathbb{R}}\left|\partial_{x} u \| \partial_{x}^{2} u\right| d x \\
& \leq 2\left\|\partial_{x} u(t, \cdot)\right\|_{L^{2}(\mathbb{R})}\left\|\partial_{x}^{2} u(t, \cdot)\right\|_{L^{2}(\mathbb{R})} \leq C .
\end{aligned}
$$

Hence,

$$
\left\|\partial_{x} u\right\|_{L^{\infty}((0, T) \times \mathbb{R})}^{2} \leq C,
$$

which gives (2.46).

Lemma 2.6 Assume that one within (1.3), (1.4), (1.5), (1.6) holds. There exist a constant $C>0$, such that

$$
\int_{0}^{t}\left\|\partial_{t} u(s, \cdot)\right\|_{L^{2}(\mathbb{R})}^{2} d s \leq C,
$$

for every $0 \leq t \leq T$. 
Proof Let $0 \leq t \leq T$. Multiplying (1.1) by $2 \partial_{t} u$, an integration on $\mathbb{R}$ gives

$$
\begin{aligned}
2\left\|\partial_{t} u(t, \cdot)\right\|_{L^{2}(\mathbb{R})}^{2}= & -2 \kappa \int_{\mathbb{R}} u \partial_{x} u \partial_{t} u d x-2 v \int_{\mathbb{R}} \partial_{x}^{2} u \partial_{t} u d x-2 \delta \int_{\mathbb{R}} \partial_{x}^{3} u \partial_{t} u d x \\
& -2 \beta^{2} \int_{\mathbb{R}} \partial_{x}^{4} u \partial_{t} u d x-2 \gamma \int_{\mathbb{R}}\left(\partial_{x} u\right)^{2} \partial_{t} u d x-2 \alpha \int_{\mathbb{R}} u \partial_{x}^{2} u \partial_{t} u d x .
\end{aligned}
$$

Therefore,

$$
\begin{aligned}
2\left\|\partial_{t} u(t, \cdot)\right\|_{L^{2}(\mathbb{R})}^{2}= & -2 \kappa \int_{\mathbb{R}} u \partial_{x} u \partial_{t} u d x-2 v \int_{\mathbb{R}} \partial_{x}^{2} u \partial_{t} u d x-2 \delta \int_{\mathbb{R}} \partial_{x}^{3} u \partial_{t} u d x \\
& -2 \beta^{2} \int_{\mathbb{R}} \partial_{x}^{4} u \partial_{t} u d x-2 \gamma \int_{\mathbb{R}}\left(\partial_{x} u\right)^{2} \partial_{t} u d x-2 \alpha \int_{\mathbb{R}} u \partial_{x}^{2} u \partial_{t} u d x .
\end{aligned}
$$

Due to the (2.2), (2.3), (2.45) and the Young inequality,

$$
\begin{aligned}
& 2|\kappa| \int_{\mathbb{R}}|u|\left|\partial_{x} u\left\|\partial_{t} u|d x \leq 2| \kappa\left|\|u\|_{L^{\infty}((0, T) \times \mathbb{R})} \int_{\mathbb{R}}\right| \partial_{x} u\right\| \partial_{t} u\right| d x \\
& \leq 2 C \int_{\mathbb{R}}\left|\partial_{x} u\right|\left|\partial_{t} u\right| d x \leq 2 \int_{\mathbb{R}}\left|\frac{C \partial_{x} u}{\sqrt{D_{1}}}\right|\left|\sqrt{D_{1}} \partial_{t} u\right| d x \\
& \leq \frac{C}{D_{1}}\left\|\partial_{x} u(t, \cdot)\right\|_{L^{2}(\mathbb{R})}^{2}+D_{1}\left\|\partial_{t} u(t, \cdot)\right\|_{L^{2}(\mathbb{R})}^{2} \\
& \leq \frac{C}{D_{1}}+D_{1}\left\|\partial_{t} u(t, \cdot)\right\|_{L^{2}(\mathbb{R})}^{2}, \\
& 2|v| \int_{\mathbb{R}}\left|\partial_{x}^{2} u \| \partial_{t} u\right| d x=2 \int_{\mathbb{R}}\left|\frac{v \partial_{x}^{2} u}{\sqrt{D_{1}}}\right|\left|\sqrt{D_{1}} \partial_{t} u\right| d x \\
& \leq \frac{v^{2}}{D_{1}}\left\|\partial_{x}^{2} u(t, \cdot)\right\|_{L^{2}(\mathbb{R})}^{2}+D_{1}\left\|\partial_{x} u(t, \cdot)\right\|_{L^{2}(\mathbb{R})}^{2} \\
& \leq \frac{C}{D_{1}}+D_{1}\left\|\partial_{x} u(t, \cdot)\right\|_{L^{2}(\mathbb{R})}^{2}, \\
& 2|\delta| \int_{\mathbb{R}}\left|\partial_{x}^{3} u\right|\left|\partial_{t} u\right| d x=2 \int_{\mathbb{R}}\left|\frac{\delta \partial_{x}^{3} u}{\sqrt{D_{1}}}\right|\left|\sqrt{D_{1}} \partial_{t} u\right| d x \\
& \leq \frac{\delta^{2}}{D_{1}}\left\|\partial_{x}^{3} u(t, \cdot)\right\|_{L^{2}(\mathbb{R})}^{2}+D_{1}\left\|\partial_{t} u(t, \cdot)\right\|_{L^{2}(\mathbb{R})}^{2}, \\
& 2 \beta^{2} \int_{\mathbb{R}}\left|\partial_{x}^{4} u\right|\left|\partial_{t} u\right| d x=2 \int_{\mathbb{R}}\left|\frac{\beta^{2} \partial_{x}^{4} u}{\sqrt{D_{1}}}\right|\left|\sqrt{D_{1}} \partial_{t} u\right| d x \\
& \leq \frac{\beta^{4}}{D_{1}}\left\|\partial_{x}^{4} u(t, \cdot)\right\|_{L^{2}(\mathbb{R})}^{2}+D_{1}\left\|\partial_{t} u(t, \cdot)\right\|_{L^{2}(\mathbb{R})}^{2}, \\
& 2|\gamma| \int_{\mathbb{R}}\left(\partial_{x} u\right)^{2}\left|\partial_{t} u\right| d x=2 \int_{\mathbb{R}}\left|\frac{\gamma\left(\partial_{x} u\right)^{2}}{\sqrt{D_{1}}}\right|\left|\sqrt{D_{1}} \partial_{t} u\right| d x \\
& \leq \frac{\gamma^{2}}{D_{1}}\left\|\partial_{x} u(t, \cdot)\right\|_{L^{4}(\mathbb{R})}^{4}+D_{1}\left\|\partial_{t} u(t, \cdot)\right\|_{L^{2}(\mathbb{R})}^{2}, \\
& 2|\alpha| \int_{\mathbb{R}}|u|\left|\partial_{x}^{2} u\right|\left|\partial_{t} u\right| d x \leq 2|\alpha|\|u\|_{L^{\infty}((0, T) \times \mathbb{R})} \int_{\mathbb{R}}\left|\partial_{x}^{2} u \| \partial_{t} u\right| d x \\
& \leq 2 C \int_{\mathbb{R}}\left|\partial_{x}^{2} u\right|\left|\partial_{t} u\right| d x \leq 2 \int_{\mathbb{R}}\left|\frac{C \partial_{x}^{2} u}{\sqrt{D_{1}}}\right|\left|\sqrt{D_{1}} \partial_{t} u\right| d x
\end{aligned}
$$




$$
\begin{aligned}
& \leq \frac{C}{D_{1}}\left\|\partial_{x}^{2} u(t, \cdot)\right\|_{L^{2}(\mathbb{R})}^{2}+D_{1}\left\|\partial_{t} u(t, \cdot)\right\|_{L^{2}(\mathbb{R})}^{2} \\
& \leq \frac{C}{D_{1}}+D_{1}\left\|\partial_{t} u(t, \cdot)\right\|_{L^{2}(\mathbb{R})}^{2},
\end{aligned}
$$

where $D_{1}$ is a positive constant, which will specified later. Consequently, by (2.49),

$$
\begin{aligned}
2\left(1-3 D_{1}\right)\left\|\partial_{t} u(t, \cdot)\right\|_{L^{2}(\mathbb{R})}^{2} \leq & \frac{C}{D_{1}}+\frac{\delta^{2}}{D_{1}}\left\|\partial_{x}^{3} u(t, \cdot)\right\|_{L^{2}(\mathbb{R})}^{2} \\
& +\frac{\beta^{4}}{D_{1}}\left\|\partial_{x}^{4} u(t, \cdot)\right\|_{L^{2}(\mathbb{R})}^{2}+\frac{\gamma^{2}}{D_{1}}\left\|\partial_{x} u(t, \cdot)\right\|_{L^{4}(\mathbb{R})}^{4} .
\end{aligned}
$$

Taking $D_{1}=\frac{1}{4}$, we have that

$$
\begin{array}{rl}
\frac{1}{2}\left\|\partial_{t} u(t, \cdot)\right\|_{L^{2}(\mathbb{R}) \leq}^{2} & C+4 \delta^{2}\left\|\partial_{x}^{3} u(t, \cdot)\right\|_{L^{2}(\mathbb{R})}^{2} \\
& +4 \beta^{4}\left\|\partial_{x}^{4} u(t, \cdot)\right\|_{L^{2}(\mathbb{R})}^{2}+4 \gamma^{2}\left\|\partial_{x} u(t, \cdot)\right\|_{L^{4}(\mathbb{R})}^{4} .
\end{array}
$$

Integrating on $(0, t)$, by (2.5), (2.6) and (2.45), we get

$$
\begin{aligned}
\frac{1}{2} \int_{0}^{t}\left\|\partial_{t} u(s, \cdot)\right\|_{L^{2}(\mathbb{R})}^{2} d s \leq & C t+4 \delta^{2} \int_{0}^{t}\left\|\partial_{x}^{3} u(s, \cdot)\right\|_{L^{2}(\mathbb{R})}^{2} d s \\
& +4 \beta^{4} \int_{0}^{t}\left\|\partial_{x}^{4} u(s, \cdot)\right\|_{L^{2}(\mathbb{R})}^{2} d s+4 \gamma^{2} \int_{0}^{t}\left\|\partial_{x} u(s, \cdot)\right\|_{L^{4}(\mathbb{R})}^{4} d s \\
\leq & C,
\end{aligned}
$$

which gives (2.48).

\section{Proof of Theorem 1.1}

This section devoted to the proof of Theorem 1.1.

Proof of Theorem 1.1 Thanks to Lemmas 2.1, or 2.3, or 2.4, 2.5, 2.6 and the CauchyKovalevskaya Theorem [63], we have that $u$ is solution of (1.1) and (1.10) holds.

We prove (1.11). Let $u_{1}$ and $u_{2}$ be two solutions of (1.1), which verify (1.10), that is

$$
\begin{aligned}
& \left\{\begin{array}{cl}
\partial_{t} u_{1}+\frac{\kappa}{2} \partial_{x} u_{1}^{2}+v \partial_{x}^{2} u_{1}+\delta \partial_{x}^{3} u_{1} & \\
+\beta^{2} \partial_{x}^{4} u_{2}+\gamma\left(\partial_{x} u_{1}\right)^{2}+\alpha u_{1} \partial_{x}^{2} u_{1}, & t>0, x \in \mathbb{R}, \\
u_{1}(0, x)=u_{1,0}(x), & x \in \mathbb{R},
\end{array}\right. \\
& \left\{\begin{array}{cl}
\partial_{t} u_{2}+\frac{\kappa}{2} \partial_{x} u_{2}^{2}+v \partial_{x}^{2} u_{2}+\delta \partial_{x}^{3} u_{2} & \\
+\beta^{2} \partial_{x}^{4} u_{2}+\gamma\left(\partial_{x} u_{2}\right)^{2}+\alpha u_{2} \partial_{x}^{2} u_{2}, & t>0, x \in \mathbb{R}, \\
u_{2}(0, x)=u_{2,0}(x), & x \in \mathbb{R} .
\end{array}\right.
\end{aligned}
$$

Then, the function

$$
\omega=u_{1}-u_{2}
$$


is the solution of the following Cauchy problem:

$$
\begin{cases}\partial_{t} \omega+\frac{\kappa}{2} \partial_{x}\left(u_{1}^{2}-u_{2}^{2}\right)+v \partial_{x}^{2} \omega+\delta \partial_{x}^{3} \omega+\beta^{2} \partial_{x}^{4} \omega & \\ +\gamma\left[\left(\partial_{x} u_{1}\right)^{2}-\left(\partial_{x} u_{2}\right)^{2}\right]+\alpha\left(u_{1} \partial_{x}^{2} u_{1}-u_{2} \partial_{x}^{2} u_{2}\right)=0, & t>0, x \in \mathbb{R}, \\ \omega(0, x)=u_{1,0}(x)-u_{2,0}(x), & x \in \mathbb{R} .\end{cases}
$$

Observe the, thanks to (3.1),

$$
u_{1} \partial_{x}^{2} u_{1}-u_{2} \partial_{x}^{2} u_{2}=u_{1} \partial_{x}^{2} u_{1}-u_{2} \partial_{x}^{2} u_{1}+u_{2} \partial_{x}^{2} u_{1}-u_{2} \partial_{x}^{2} u_{2}=\omega \partial_{x}^{2} u_{1}+u_{2} \partial_{x}^{2} \omega .
$$

Therefore, (3.2) reads

$$
\begin{aligned}
\partial_{t} \omega & +\frac{\kappa}{2} \partial_{x}\left(u_{1}^{2}-u_{2}^{2}\right)+v \partial_{x}^{2} \omega+\delta \partial_{x}^{3} \omega+\beta^{2} \partial_{x}^{4} \omega \\
& +\gamma\left[\left(\partial_{x} u_{1}\right)^{2}-\left(\partial_{x} u_{2}\right)^{2}\right]+\alpha \omega \partial_{x}^{2} u_{1}+\alpha u_{2} \partial_{x}^{2} \omega=0 .
\end{aligned}
$$

Since, thanks to (3.1),

$$
\begin{aligned}
2 \int_{\mathbb{R}} \omega \partial_{t} \omega d x & =\frac{d}{d t}\|\omega(t, \cdot)\|_{L^{2}(\mathbb{R})}^{2}, \\
\kappa \int_{\mathbb{R}} \partial_{x}\left(u_{1}^{2}-u_{2}^{2}\right) \omega & =\kappa \int_{\mathbb{R}}\left(u_{1}+u_{2}\right) \omega \partial_{x} \omega d x, \\
2 v \int_{\mathbb{R}} \omega \partial_{x}^{2} \omega & =-2 v\left\|\partial_{x} \omega(t, \cdot)\right\|_{L^{2}(\mathbb{R})}^{2}, \\
2 \delta \int_{\mathbb{R}} \omega \partial_{x}^{3} \omega d x & =-2 \delta \int_{\mathbb{R}} \partial_{x} \omega \partial_{x}^{2} \omega d x=0, \\
2 \beta^{2} \int_{\mathbb{R}} \omega \partial_{x}^{4} \omega & =-2 \beta \int_{\mathbb{R}} \partial_{x} \omega \partial_{x}^{3} \omega d x=2 \beta^{2}\left\|\partial_{x}^{2} \omega(t, \cdot)\right\|_{L^{2}(\mathbb{R})}^{2}, \\
2 \gamma \int_{\mathbb{R}}\left[\left(\partial_{x} u_{1}\right)^{2}-\left(\partial_{x} u_{2}\right)^{2}\right] \omega d x & =2 \gamma \int_{\mathbb{R}}\left(\partial_{x} u_{1}+\partial_{x} u_{2}\right) \omega \partial_{x} \omega d x,
\end{aligned}
$$

multiplying (3.2) by $2 \omega$, an integration on $\mathbb{R}$ gives

$$
\begin{aligned}
\frac{d}{d t} \| & \omega(t, \cdot)\left\|_{L^{2}(\mathbb{R})}^{2}+2 \beta^{2}\right\| \partial_{x}^{2} \omega(t, \cdot) \|_{L^{2}(\mathbb{R})}^{2} \\
= & -\kappa \int_{\mathbb{R}}\left(u_{1}+u_{2}\right) \omega \partial_{x} \omega d x+2 v\left\|\partial_{x} \omega(t, \cdot)\right\|_{L^{2}(\mathbb{R})}^{2} \\
& -2 \gamma \int_{\mathbb{R}}\left(\partial_{x} u_{1}+\partial_{x} u_{2}\right) \omega \partial_{x} \omega d x+2 \alpha \int_{\mathbb{R}} \omega^{2} \partial_{x}^{2} u_{1} d x \\
& +2 \alpha \int_{\mathbb{R}} u_{2} \omega \partial_{x}^{2} \omega d x .
\end{aligned}
$$

Observe that, since $u_{1}, u_{2} \in H^{2}(\mathbb{R})$, for every $0 \leq t \leq T$, we have that

$$
\begin{aligned}
& \left\|u_{1}\right\|_{L^{\infty}((0, T) \times \mathbb{R})},\left\|\partial_{x} u_{1}\right\|_{L^{\infty}((0, T) \times \mathbb{R})} \leq C, \\
& \left\|u_{2}\right\|_{L^{\infty}((0, T) \times \mathbb{R})},\left\|\partial_{x} u_{2}\right\|_{L^{\infty}((0, T) \times \mathbb{R})} \leq C, \\
& \left\|\partial_{x}^{2} u_{1}(t, \cdot)\right\|_{L^{2}(\mathbb{R})}^{2} \leq C .
\end{aligned}
$$

Consequently, thanks to (3.5), we obtain that

$$
\left|u_{1}+u_{2}\right| \leq C, \quad\left|\partial_{x} u_{1}+\partial_{x} u_{2}\right| \leq C .
$$


Due to (3.5), (3.6) and the Young inequality,

$$
\begin{aligned}
& |\kappa| \int_{\mathbb{R}}\left|u_{1}+u_{2}\right||\omega|\left|\partial_{x} \omega\right| d x \leq C(T) \int_{\mathbb{R}}|\omega| \partial_{x} \omega \mid d x \\
& \quad \leq C\|\omega(t, \cdot)\|_{L^{2}(\mathbb{R})}^{2}+C\left\|\partial_{x} \omega(t, \cdot)\right\|_{L^{2}(\mathbb{R})}^{2}, \\
& 2|\gamma| \int_{\mathbb{R}}\left|\partial_{x} u_{1}+\partial_{x} u_{2}\right||\omega|\left|\partial_{x} \omega\right| d x \leq C \int_{\mathbb{R}}|\omega| \partial_{x} \omega \mid d x \\
& \quad \leq C\|\omega(t, \cdot)\|_{L^{2}(\mathbb{R})}^{2}+C\left\|\partial_{x} \omega(t, \cdot)\right\|_{L^{2}(\mathbb{R})}^{2}, \\
& 2|\alpha| \int_{\mathbb{R}} \omega^{2}\left|\partial_{x}^{2} u_{1}\right| d x \leq\|\omega(t, \cdot)\|_{L^{2}(\mathbb{R})}^{2}+\alpha^{2} \int_{\mathbb{R}} \omega^{2}\left(\partial_{x}^{2} u_{1}\right)^{2} d x \\
& \quad \leq\|\omega(t, \cdot)\|_{L^{2}(\mathbb{R})}^{2}+\alpha^{2}\|\omega(t, \cdot)\|_{L^{\infty}(\mathbb{R})}^{2}\left\|\partial_{x}^{2} u_{1}(t, \cdot)\right\|_{L^{2}(\mathbb{R})}^{2} \\
& \quad \leq\|\omega(t, \cdot)\|_{L^{2}(\mathbb{R})}^{2}+C\|\omega(t, \cdot)\|_{L^{\infty}(\mathbb{R})}^{2}, \\
& 2|\alpha| \int_{\mathbb{R}}\left|u_{2}\left\|\omega|| \partial_{x}^{2} \omega|d x \leq 2| \alpha\left|\left\|u_{2}\right\|_{L^{\infty}((0, T) \times \mathbb{R})} \int_{\mathbb{R}}\right| \omega\right\| \partial_{x}^{2} \omega\right| d x \\
& \quad \leq 2 C \int_{\mathbb{R}}\left|\omega \| \partial_{x}^{2} \omega\right| d x=2 \int_{\mathbb{R}}\left|\frac{C(T) \omega}{\beta}\right| \beta \partial_{x}^{2} \omega \mid d x \\
& \quad \leq C\|\omega(t, \cdot)\|_{L^{2}(\mathbb{R})}^{2}+\beta^{2}\left\|\partial_{x}^{2} \omega(t, \cdot)\right\|_{L^{2}(\mathbb{R})}^{2} \cdot
\end{aligned}
$$

It follows from (3.4) that

$$
\begin{aligned}
& \frac{d}{d t}\|\omega(t, \cdot)\|_{L^{2}(\mathbb{R})}^{2}+2 \beta^{2}\left\|\partial_{x}^{2} \omega(t, \cdot)\right\|_{L^{2}(\mathbb{R})}^{2} \\
& \quad \leq C\|\omega(t, \cdot)\|_{L^{2}(\mathbb{R})}^{2}+C\left\|\partial_{x} \omega(t, \cdot)\right\|_{L^{2}(\mathbb{R})}^{2}+C\|\omega(t, \cdot)\|_{L^{\infty}(\mathbb{R})}^{2} .
\end{aligned}
$$

Observe that, by the Hölder inequality,

$$
\omega^{2}(t, x)=2 \int_{-\infty}^{x} \omega \partial_{x} \omega d y \leq 2 \int_{\mathbb{R}}|\omega|\left|\partial_{x} \omega\right| d x \leq 2\|\omega(t, \cdot)\|_{L^{2}(\mathbb{R})}\left\|\partial_{x} \omega(t, \cdot)\right\|_{L^{2}(\mathbb{R})} .
$$

Hence,

$$
\|\omega(t, \cdot)\|_{L^{2}(\mathbb{R})}^{2} \leq 2\|\omega(t, \cdot)\|_{L^{2}(\mathbb{R})}\left\|\partial_{x} \omega(t, \cdot)\right\|_{L^{2}(\mathbb{R})} .
$$

Due to the Young inequality,

$$
\|\omega(t, \cdot)\|_{L^{2}(\mathbb{R})}^{2} \leq\|\omega(t, \cdot)\|_{L^{2}(\mathbb{R})}^{2}+\left\|\partial_{x} \omega(t, \cdot)\right\|_{L^{2}(\mathbb{R})}^{2} .
$$

Consequently, by (3.7),

$$
\begin{aligned}
\frac{d}{d t}\|\omega(t, \cdot)\|_{L^{2}(\mathbb{R})}^{2} & +\beta^{2}\left\|\partial_{x}^{2} \omega(t, \cdot)\right\|_{L^{2}(\mathbb{R})}^{2} \\
& \leq C\|\omega(t, \cdot)\|_{L^{2}(\mathbb{R})}^{2}+C\left\|\partial_{x} \omega(t, \cdot)\right\|_{L^{2}(\mathbb{R})}^{2} .
\end{aligned}
$$

Observe that

$$
C\left\|\partial_{x} \omega(t, \cdot)\right\|_{L^{2}(\mathbb{R})}^{2}=C \int_{\mathbb{R}} \partial_{x} \omega \partial_{x} \omega d x=-C \int_{\mathbb{R}} \omega \partial_{x}^{2} \omega d x
$$

Therefore, by the Young inequality,

$$
C\left\|\partial_{x} \omega(t, \cdot)\right\|_{L^{2}(\mathbb{R})}^{2} \leq 2 \int_{\mathbb{R}}\left|\frac{C \omega}{2 \beta}\right|\left|\beta \partial_{x}^{2} \omega\right| d x
$$




$$
\leq C\|\omega(t, \cdot)\|_{L^{2}(\mathbb{R})}^{2}+\frac{\beta^{2}}{2}\left\|\partial_{x}^{2} \omega(t, \cdot)\right\|_{L^{2}(\mathbb{R})}^{2} \cdot
$$

It follows from (3.9) that

$$
\frac{d}{d t}\|\omega(t, \cdot)\|_{L^{2}(\mathbb{R})}^{2}+\frac{\beta^{2}}{2}\left\|\partial_{x}^{2} \omega(t, \cdot)\right\|_{L^{2}(\mathbb{R})}^{2} \leq C\|\omega(t, \cdot)\|_{L^{2}(\mathbb{R})}^{2} .
$$

The Gronwall Lemma and (3.2) gives

$$
\|\omega(t, \cdot)\|_{L^{2}(\mathbb{R})}^{2}+\frac{\beta^{2} e^{C t}}{2} \int_{0}^{t} e^{-C s}\left\|\partial_{x}^{2} \omega(s, \cdot)\right\|_{L^{2}(\mathbb{R})}^{2} d s \leq e^{C t}\left\|\omega_{0}\right\|_{L^{2}(\mathbb{R})}^{2} .
$$

(1.10) follows from (3.1) and (3.10).

Funding Open access funding provided by Politecnico di Bari within the CRUI-CARE Agreement.

\section{Declarations}

Conflict of interest The authors declare that they have no conflict of interest.

Open Access This article is licensed under a Creative Commons Attribution 4.0 International License, which permits use, sharing, adaptation, distribution and reproduction in any medium or format, as long as you give appropriate credit to the original author(s) and the source, provide a link to the Creative Commons licence, and indicate if changes were made. The images or other third party material in this article are included in the article's Creative Commons licence, unless indicated otherwise in a credit line to the material. If material is not included in the article's Creative Commons licence and your intended use is not permitted by statutory regulation or exceeds the permitted use, you will need to obtain permission directly from the copyright holder. To view a copy of this licence, visit http://creativecommons.org/licenses/by/4.0/.

\section{References}

1. Christov, C.I., Velarde, M.G.: Dissipative solitons. Phys. D 86(32), 323-347 (1995)

2. Garcia-Ybarra, P.L., Castillo, J.L., Velarde, M.G.: Benard-Marangoni convection with a deformable interface and poorly conducting boundaries. Phys. Fluids 30, 2655-2661 (1987)

3. Garcia-Ybarra, P.L., Castillo, J.L., Velarde, M.G.: A nonlinear evolution equation for Benard-Marangoni convection with deformable boundary. Phys. Lett. A 122, 107-110 (1987)

4. Hyman, J.M., Nicolaenko, B.: Coherence and chaos in Kuramoto-Velarde equation. In: Grandall, M.G., Rabinovitz, P.H., Turner, R.E.L. (eds.) Directions in Partial Differential Equations, pp. 89-111. Academic Press, New York (1987)

5. Velarde, M.G., Normand, C.: Convection. Sci. Am. 243, 92-108 (1980)

6. Zierep Jr., J., Oertel, H.: Convective Transport and Instability Phenomena. Braun, Karlsruhe (1982)

7. Topper, J., Kawahara, T.: Approximate equations for long nonlinear waves on a viscous fluid. J. Phys. Soc. Jpn 44, 663-666 (1978)

8. Cohen, B.I., Krommes, J.A., Tang, W.M., Rosenbluth, M.N.: Nonlinear saturation of the dissipative trapped-ion mode by mode coupling. Nuclear Fus. 16, 971-992 (1976)

9. Coclite, G.M., di Ruvo, L.: The well-posedness of a hight order convective Cahn-Hilliard type equation. Algorithms 13(7), 170 (2020)

10. Coclite, G.M., di Ruvo, L.: A note on the solutions for a higher order convective Cahn-Hilliard type equation. Mathematics 8, 1835 (2020)

11. Coclite, G.M., di Ruvo, L.: Well-posedness of the classical solution for the Kuramoto-Sivashinsky equation with anisotropy effects. Z. Angew. Math. Phys. 72, 1-37 (2021)

12. Emmott, C.L., Bray, A.J.: Coarsening dynamics of a one-dimensional driven Cahn-Hilliard system. Phys. Rev. E 54, 4568 (1996)

13. Leung, K.: Theory on morphological instability in driven systems. J. Stat. Phys. 61, 345 (1990) 
14. Yeung, C., Rogers, T., Hernandes-Machado, A., Jasnow, D.: Phase separation dynamics in driven diffusive systems. J. Stat. Phys. 66, 1071 (1992)

15. Golovin, A.A., Davis, S.H., Nepomnyashchy, A.A.: A convective Cahn-Hilliard model for the formation of facets and comers in crystal growth. Phys. D 122, 202-230 (1998)

16. Khalique, C.M.: Exact solutions of the generalized Kuramoto-Sivashinsky equation. Caspian J. Math. Sci. 1(2), 109-116 (2012)

17. Saito, Y., Uwaha, M.: Anisotropy effect on step morphology described by Kuramoto-Sivashinsky equation. J. Phys. Soc. Jpn. 65, 3576-3581 (1996)

18. Golovin, A.A., Davis, S.H., Nepomnyashchy, A.A.: Model for faceting in a kinetically controlled crystal growth. Phys. Rev. E 59, 803 (1999)

19. Golovin, A.A., Nepomnyashchy, A.A., Davis, S.H., Zaks, M.A.: Convective Cahn-Hilliard models: from coarsening to roughening. Phys. Rev. Lett. 86, 1550-1553 (2001)

20. Gurtin, M.E.: Thermomechanics of evolving phase boundaries in the plane. Clarendon Press, Oxford (1993)

21. Liu, F., Metiu, H.: Dynamics of phase separation of crystal surfaces. Phys. Rev. B 48(9), 5808 (1993)

22. Kamenov, O.Y.: Solitary-wave and periodic solutions of the Kuramoto-Velarde dispersive equation. J. Theor. Appl. Mech. 46, 65-74 (2016)

23. Rodríguez-Bernal, A.: Initial value problem and asymptotic low-dimensional behavior in the KuramotoVelarde equation. Nonlinear Anal. Theory Methods Appl. 19, 643-685 (1992)

24. Christov, C.I., Velarde, M.G.: On localized solutions of an equation governing Bénard-Marangoni convection. Appl. Math. Model. 17, 311-320 (1993)

25. Mansour, M.B.A.: Existence of traveling wave solutions for a nonlinear dissipative-dispersive equation. Appl. Math. Mech. Engl. Ed. 30(4), 513-516 (2009)

26. Kamenov, O.Y.: Periodic solutions of the non-integrable convective fluid equation. J. Math. Phys. 53, 063705 (2012)

27. Pilod, D.: Sharp well-posedness results for the Kuramoto-Velarde equation. Commun. Pure Appl. Anal. 7, 867-881 (2008)

28. Coclite, G.M., di Ruvo, L.: On the classical solutions for a Kuramoto-Sinelshchikov-Velarde type equation. Algorithms 13(4), 1-22 (2020)

29. Kuramoto, Y.: Diffusion-induced Chaos in reaction systems. Prog. Theor. Phys. Suppl. 64, 346-367 (1978)

30. Kuramoto, Y., Tsuzuki, T.: On the formation of dissipative structures in reaction-diffusion systems: reductive perturbation approach. Prog. Theor. Phys. 54, 687-699 (1975)

31. Kuramoto, Y., Tsuzuki, T.: Persistent propagation of concentration waves in dissipative media far from thermal equilibrium. Prog. Theor. Phys. 55, 356-369 (1976)

32. Sivashinsky, G.: Nonlinear analysis of hydrodynamic instability in laminar flames-I. Derivation of basic equations. Acta Astronaut. 4, 1177-1206 (1977)

33. Chen, L.H., Chang, H.C.: Nonlinear waves on liquid film surfaces-II. Bifurcation analyses of the longwave equation. Chem. Eng. Sci. 41, 2477-2486 (1986)

34. Hooper, A.P., Grimshaw, R.: Nonlinear instability at the interface between two viscous fluids. Phys. Fluids 28, 37-45 (1985)

35. Laquey, R.E., Mahajan, S.M., Rutherford, P.H.: Nonlinear saturation of the trapped-ion mode. Phys. Rev. Lett. 34, 391-394 (1975)

36. Benney, D.J.: Long waves on liquid films. J. Math. Phys. 45, 150-155 (1966)

37. Lin, S.P.: Finite amplitude side-band stability of a viscous film. J. Fluid Mech. 63(3), 417-429 (1974)

38. Li, C., Chen, G., Zhao, S.: Exact travelling wave solutions to the generalized Kuramoto-Sivashinsky equation. Lat. Am. Appl. Res. 34, 64-68 (2004)

39. Foias, C., Nicolaenko, B., Sell, G.R., Temam, R.: Inertial manifolds for the Kuramoto-Sivashinsky equation and an estimate of their lowest dimension. J. Math. Pures Appl. 67, 197-226 (1988)

40. Khenner, M.: Long-wave model for strongly anisotropic growth of a crystal step. Phys. Rev. E 88, 022402 (2013)

41. Kudryashov, N.A.: Exact solutions of the generalized Kuramoto-Sivashinsky equation. Phys. Lett. A 147, 287-291 (1990)

42. Nicolaenko, B., Scheurer, B.: Remarks on the Kuramoto-Sivashinsky equation. Phys. D 12, 391-395 (1984)

43. Nicolaenko, B., Scheurer, B., Temam, R.: Some global dynamical properties of the Kuramoto-Sivashinsky equations: nonlinear stability and attractors. Phys. D 16, 155-183 (1985)

44. Xie, Y.: Solving the generalized Benney equation by a combination method. I. J. Nonlinear Sci. 15(4), 350-354 (2013) 
45. Armaou, A., Christofides, P.D.: Feedback control of the Kuramoto-Sivashinsky equation. Phys. D 137, 49-61 (2000)

46. Cerpa, E.: Null controllability and stabilization of the linear Kuramoto-Sivashinsky equation. Commun. Pure Appl. Anal. 9, 91-102 (2010)

47. Giacomelli, L., Otto, F.: New bounds for the Kuramoto-Sivashinsky equation. Commun. Pure Appl. Math. 58, 297-318 (2005)

48. Christofides, P.D., Armaou, A.: Global stabilization of the Kuramoto-Sivashinsky equation via distributed output feedback control. Syst. Control Lett. 39, 283-294 (2000)

49. Hu, C., Temam, R.: Robust control of the Kuramoto-Sivashinsky equation. Dyn. Contin. Discrete Impuls. Syst. Ser. B Appl. Algorithms 8, 315-338 (2001)

50. Liu, W.J., Krstic, M.: Stability enhancement by boundary control in the Kuramoto-Sivashinsky equation. Nonlinear Anal. 43, 485-507 (2001)

51. Sajjadian, M.: The shock profile wave of Kuramoto-Sivashinsky equation and solitonic solution of generalized Kuramoto-Sivashinsky equation. Acta Univ. Apulensis Mat. Inform. 38, 163-176 (2014)

52. Biagioni, H.A., Bona, J.L., Iorio, R., Scialom, M.: On the Korteweg-de Vries-Kuramoto-Sivashinsky equation. Adv. Differ. Equ. 1, 1-20 (1996)

53. Tadmor, E.: The well-posedness of the Kuramoto-Sivashinsky equation. SIAM J. Math. Anal. 17(4), 884-893 (1986)

54. Coclite, G. M., di Ruvo, L.: $H^{1}$ solutions a Kuramoto-Sinelshchikov-Cahn-Hilliard type equation. Ric. Mat (to appear)

55. Coclite, G.M., di Ruvo, L.: On the initial-boundary value problem for a Kuramoto-Sinelshchikov type equation. Math. Eng. 3(4), 1-43 (2020)

56. Li, J., Zhang, B.Y., Zhang, Z.: A nonhomogeneous boundary value problem for the Kuramoto-Sivashinsky equation in a quarter plane. Math. Methods Appl. Sci. 40(15), 5619-5641 (2017)

57. Li, J., Zhang, B.Y., Zhang, Z.: A non-homogeneous boundary value problem for the KuramotoSivashinsky equation posed in a finite interval. ESAIM: COCV 26, 43 (2020)

58. Coclite, G.M., di Ruvo, L.: Dispersive and diffusive limits for Ostrovsky-Hunter type equations. Nonlinear Differ. Equ. Appl. 22, 1733-1763 (2015)

59. LeFloch, P.G., Natalini, R.: Conservation laws with vanishing nonlinear diffusion and dispersion. Nonlinear Anal. Ser. A Theory Methods 36(2), 212-230 (1992)

60. Schonbek, M.E.: Convergence of solutions to nonlinear dispersive equations. Comm. Partial Differ. Equ. 7(8), 959-1000 (1982)

61. Coclite, G.M., di Ruvo, L.: Convergence of the Kuramoto-Sinelshchikov equation to the Burgers one. Acta Appl. Math. 145(1), 89-113 (2016)

62. Coclite, G.M., di Ruvo, L.: Existence results for the Kudrayashov-Sinelshchikov-Olver equation. Proc. R. Soc. Edinburgh Sect. A. 151, 1-26 (2020)

63. Taylor, M.E.: Partial Differential Equations I: Basic Theory, 2nd edn. Springer, New York (2011)

Publisher's Note Springer Nature remains neutral with regard to jurisdictional claims in published maps and institutional affiliations. 